\title{
Achieving photo-control of protein conformation and activity: producing a photo-controlled leucine zipper
}

\author{
Janet R. Kumita, ${ }^{a}$ Daniel G. Flint, ${ }^{b}$ G. Andrew Woolley ${ }^{a}$ and Oliver S. Smart ${ }^{b}$ \\ a Department of Chemistry, The University of Toronto, 80 St. George Street, Toronto \\ M5S 3H6, Canada \\ ${ }^{b}$ School of Biosciences, The University of Birmingham, Edgbaston, Birmingham, UK \\ B15 2TT. E-mail: o.s.smart@bham.ac.uk
}

Received 24th January 2002, Accepted 1st March 2002

First published as an Advance Article on the web 17th July 2002

We have recently developed a technique that has great potential in producing proteins with photo-control of conformation and consequently activity (J. R. Kumita, O. S. Smart and G. A. Woolley, Proc. Natl. Acad. Sci. U. S. A., 2000, 97, 3803-3808). The method is based on incorporating two cysteine residues into the sequence of a polypeptide. An azobenzene derivative is subsequently used to produce an intramolecular cross-link between the cysteine sulfhydryl groups. In previous work photo-isomerisation of the azobenzene moiety has been used to control the helicity of a monomeric peptide. In the experiments described here this method has been applied to the coiled coil leucine zipper peptide GCN4-p1. The aim was to produce a variant of GCN4-p1 whose helicity and consequently dimerisation is under direct photo-control. We have produced a modified GCN4-p1 incorporating two cysteine residues. The mutations introduced are shown to interfere with the ability of the uncross-linked peptide to form a coiled coil. After the peptide was cross-linked with the azobenzene derivative more normal coiled-coil behaviour was restored. Irradiation of the peptide producing a conformational change in the azobenzene cross-linker was accompanied by an increase in the helicity of the peptide. The work presented here highlights the potential of the use of photo-isomerisable cross-linkers to control protein activity through induced conformational change. In addition, the methodology has the potential to provide a fast trigger for the initiation of protein conformational changes.

\section{Introduction}

The selective control of the activity of a protein through the application of an external source of light has many potential applications. ${ }^{1}$ Photo-isomerisable chromophores are most suitable in this area as they introduce the possibility of rapid yet reversible control. Work to date has concentrated on the selective introduction of photo-isomerisable compounds, in particular azobenzene, into peptides. The azobenzene chromophore has been introduced into the backbone of cyclic $\beta$-turn peptides by Moroder, Chmielewski and co-workers. ${ }^{2-5}$ Others have investigated the effect of amino acids with azobenzene side chains on biological systems. ${ }^{6,7}$ In general these studies, although encouraging, have not led to a general and easily applied method of achieving photo-control of the conformation and activity of a protein. 
A<smiles>O=C(CI)Nc1ccc(/N=N/c2ccc(NC(=O)CI)cc2)cc1</smiles><smiles>CC(C)[AlH2]</smiles><smiles>O=C(CI)Nc1ccc(/N=N\c2ccc(NC(=O)CI)cc2)cc1</smiles>

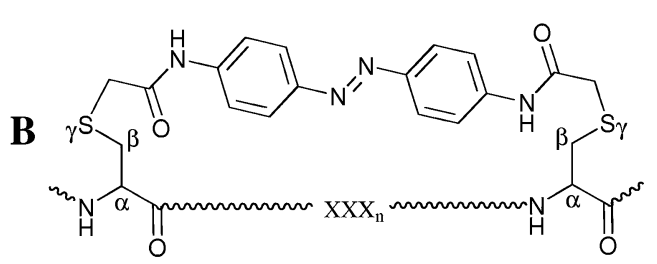

Fig. 1 The structure of the azobenzene-based cross-linker. (A) Chemical structure ${ }^{8}$ of the azobenzene crosslinker 1 showing how it can be photo-isomerised. (B) How the compound 1 can cross-link a pair of cysteine residues within a peptide or protein.

In recent work we have developed an alternative approach ${ }^{8}$ which has great promise. The method employs compound 1 where the photo-isomerisable azobenzene chromophore is flanked by two iodoacetamide functional groups (Fig. 1A). Compound $\mathbf{1}$ can be used to cross-link a pair of cysteine side chains within a polypeptide chain as the iodoacetamide group reacts selectively with the sulfhydryl group of the cysteine side-chain (Fig. 1B). This allows the compound to be used to cross-link either peptides that have been produced by conventional solid-phase synthesis methods or recombinant proteins.

The initial application of the cross-linker was in the photo-control of the conformation of a monomeric 16-residue peptide. ${ }^{8}$ Molecular modelling was used to design a peptide that would be $\alpha$-helical when the azobenzene cross-linker was in the cis (photo-isomerised) conformation but unfolded when the cross-linker adopted the trans (dark adapted) form. It was predicted that a relative spacing of $i$ to $i+7$ between the cysteine residues would result in this behaviour. In practice, circular dichroism spectropolarimetry $(\mathrm{CD})$ was used to show that photo-isomerisation of the cross-linker dramatically increased the helicity of the peptide. Using the CD signal at $222 \mathrm{~nm}$ as a gauge of $\alpha$-helix content, results indicate that the dark-adapted cross-linked peptide was $12 \%$ helical (at $11^{\circ} \mathrm{C}$ ). Upon illumination with $370 \mathrm{~nm}$ light (the optimum wavelength for inducing cross-linker photo-isomerisation) apparent helix content increased to $48 \%{ }^{8}$ It should be noted that a limitation of the azobenzene chromophore is that it is not possible to switch the entire population of the cross-linker to a cis conformation and that for this peptide only $77 \%$ photo-isomerisation was achieved (unpublished results; using the method set out below). When this is taken into account the percentage helicity of the peptide with the cross-linker in a cis conformation can be estimated to be around $60 \%$.

In a series of studies we have extended these initial results. In the original peptide with a cysteine spacing of $(i, i+7) \alpha$-aminoisobutyric acid (Aib), an unnatural amino acid, was incorporated under the cross-linker at the $(i+4)$ site. ${ }^{8}$ This limited the use of the cross-linker to peptides that could be synthesised by solid-phase methodology to facilitate the incorporation of Aib. However, further 
work has demonstrated that the presence of coded amino acids at this position results in conformational changes similar to those seen in the original system. ${ }^{9 a}$ This shows that the cross-linker is functionally applicable for peptides and proteins containing only coded amino acids.

Furthermore, we have shown that the cross-linker can be used with different spacings between the cysteine residues. ${ }^{9 b}$ Improved molecular modelling procedures indicated that a relative cysteine spacing of $(i, i+4)$ could be used to produce a peptide that would form an $\alpha$-helix when the crosslinker was in the cis isomeric form. A cross-linked peptide with this spacing would be unable to adopt a helical conformation with the trans isomer (similar to the original peptide). In contrast, a spacing of $(i, i+11)$ is suitable for producing a peptide that is helical when the cross-linker is in the dark-adapted trans conformation and is forced to unfold upon photo-isomerisation of the crosslinker to the cis form. Peptides incorporating these features were synthesised and shown to behave as expected. ${ }^{9}$ This knowledge will be most useful when applied to proteins, we can now choose the direction of photo-control as well as the size of the region affected.

The cross-linker has great potential in aiding time-resolved studies of protein conformational changes. The photo-isomerisation process of azobenzene compounds is fast, typically occurring ${ }^{10}$ in less than $10 \mathrm{ps}$. This means that if, for instance, the folding of a protein was coupled to the photo-controlled formation of an individual helix this methodology could provide a sensitive and selective trigger for the change. An alternative approach utilising the photolysis of an aryl disulfide cross-linker demonstrates that photo-control can be fruitfully applied as a trigger for peptide conformational change. ${ }^{11}$

In this work, we seek to apply the method to control the conformation and behaviour of a coiled coil peptide. The coiled coil is one of the most ubiquitous assembly motifs found in proteins. ${ }^{12}$ As exemplified by GCN4-p1, it normally consists of two parallel amphipathic $\alpha$-helices that wrap around each other to form a homodimeric left-handed supercoil ${ }^{13}$ (Fig. 3(A), below). This results in favourable interactions between hydrophobic residues spaced every 4 then 3 residues apart in the sequence, a pattern defined as a heptad repeat ${ }^{13}$ (abcdefg) ${ }_{n}$ (Fig. 2). Hydrophobic residues occur at positions a and d (Fig. 2B). For a family of coiled coils these hydrophobic residues are predominately leucine leading to the name leucine zipper. ${ }^{14}$ Flanking the hydrophobic core heptad repeat positions e and $\mathrm{g}$ are often occupied by charged residues that form inter-helical salt bridges ${ }^{13}$
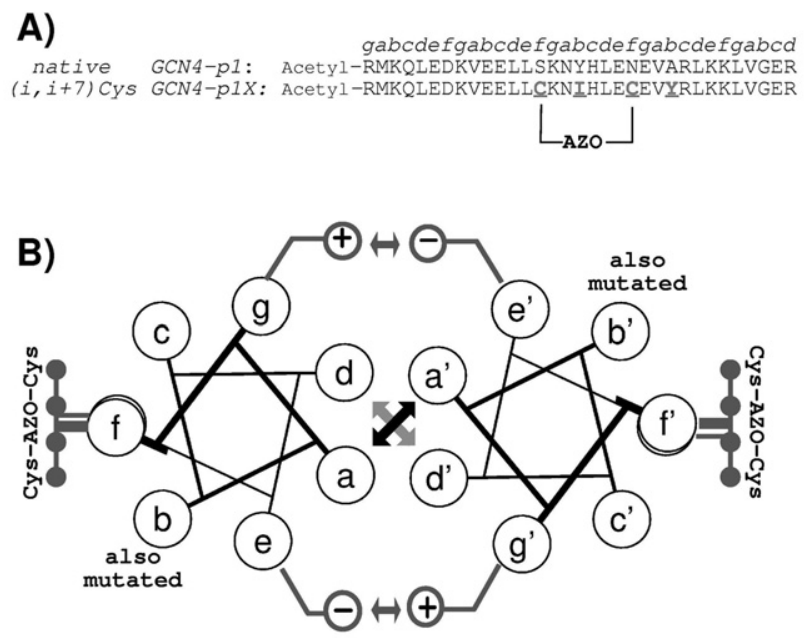

Fig. 2 The proposed cross-linked GCN4-p1. (A) The primary structure in one letter code of the modified GCN4-p1 compared to the native sequence, with the heptad repeat shown at the top. (B) How the modification fits on a helical wheel diagram for GCN4-p1. The arrows at the centre of the interface represent the hydrophobic interaction between residues in the $a$ and $d$ positions of the heptad repeat. The modifications to the native sequence are limited to the $f$ and $b$ positions of the heptad repeat and should not disrupt the dimer interface. 
(Fig. 2B). Amino acids in positions b, $\mathrm{c}$ and $\mathrm{f}$ lie on the external faces of the coil and are tolerant of amino acid variability. ${ }^{15}$

We have chosen to work with the leucine zipper ${ }^{13}$ from the yeast transcriptional activator protein GCN4. GCN4-p1 is a 33 residue peptide found at the C-terminus of the protein that forms a coiled coil. ${ }^{13}$ This peptide is attractive because it has been very widely studied and has a two-state folding mechanism from random coil monomers to folded helical dimers. ${ }^{14}$ This means that $\mathrm{CD}$ can be used as a powerful tool in the analysis of the behaviour of GCN4-p1 since the signal at $222 \mathrm{~nm}$ can generally be directly related to association. ${ }^{14,16}$

The leucine zipper GCN4-p1 is also an attractive system for photo-control as it should be possible to extend these studies to the longer (56 residue) GCN4-bZIP protein that comprises a basic DNA binding helix followed by the leucine zipper. An X-ray crystal structure of GCN4-bZIP bound to its cognate DNA shows it to be a Y-shaped dimer of two extended $\alpha$-helices. ${ }^{17}$ The zipper forms the body of the $\mathrm{Y}$ and joins the two arm-like basic regions, each of which binds into the major groove of DNA. ${ }^{17}$ By controlling the helix propensity and, as a consequence dimerisation, of the leucine zipper part of the protein we can expect a major influence on the DNA binding ability of the longer protein. This is because even small changes in the helix propensity of the zipper region can have dramatic effects on the DNA binding ability of a bZIP protein. This is elegantly demonstrated in a study conducted by Vinson and co-workers ${ }^{18}$ where the phosphorylation of a residue in the zipper region had a small effect on the percentage helicity of an isolated zipper peptide but produced a dramatic 100-fold effect on DNA binding. Our approach differs markedly from that of Mascareñas and co-workers ${ }^{19}$ who have used an azobenzene-based compound to produce an intermolecular cross-link between a pair of basic DNA binding helices taken from GCN4-bZIP. An encouraging degree of photo-control of DNA binding was found, albeit with a problem of irreversibility. ${ }^{19}$

By incorporating the cross-linker into GCN4-p1, we aim to produce a coiled coil peptide whose helicity and, consequently, dimerisation is under direct photo-control. Such a peptide would be the first example of a widely applicable methodology that could potentially allow the external control of the dimerisation of a macromolecule.

\section{Materials and methods}

\section{Synthesis of azobenzene cross-linking reagent}

The azobenzene cross-linking reagent containing two cysteine-reactive iodoacetamide groups (structure 1) was synthesised as reported previously. ${ }^{8}$

\section{Peptide design}

Peptide design was based on the high-resolution X-ray crystal structure for GCN4-p1. ${ }^{13}$ Molecular Graphics and modelling were performed using the SYBYL package (Tripos Inc) and the AMBER 4. $1^{20}$ potential energy function as described below. $\mathrm{VMD}^{21}$ and Raster3D ${ }^{22}$ were used to produce pictures of the molecular structures. A Silicon Graphics Octane2 workstation was used for all computational work.

\section{Peptide synthesis}

Standard fluorenylmethoxycarbonyl-based solid-phase peptide synthesis methods were used to prepare the modified $(i, i+7)$ Cys GCN4-p1: acetyl-RMKQLEDKVEELLCKNIHLECEVYRLKKLVGER and native GCN4-p1: Acetyl-RMKQLEDKVEELLSKNYHLENEVARLKKLVGER (Fig. 2A). The peptides were constructed on Fmoc-Arg(Pbf)-Wang resin (capacity $0.35 \mathrm{mmol} \mathrm{g}{ }^{-1}$ ) (Novabiochem, San Diego CA). Coupling used 3 equiv. HATU $\{O$-(7-azobenzotriazol-1-yl)1,1,3,3-tetramethyluronium hexafluorophosphate\} (Sigma-Aldrich, Canada), 6 equiv. DIPEA ( $N$, $N$,-diisopropylethylamine), 3 equiv. amino acid (Novabiochem, San Diego CA). Peptides were purified by HPLC (Zorbax SB-C18 column) using a linear gradient from 0 to $75 \%$ acetonitrile $/ \mathrm{H}_{2} \mathrm{O}$ $(+0.1 \%$ trifluoroacetic acid) over $50 \mathrm{~min}$ for $(i, i+7)$ Cys GCN4-p1 (eluted at $49.8 \%$ acetonitrile) and native GCN4-p1 (eluted at $46.2 \%$ acetonitrile). The peptide primary structure was confirmed by MALDI-MS for $(i, i+7)$ Cys GCN4-p1 (observed: $4083.0 \mathrm{Da}$; calculated $\left(\mathrm{C}_{178} \mathrm{H}_{302} \mathrm{~N}_{51} \mathrm{O}_{52} \mathrm{~S}_{3}\right)$ : 
4083.4 Da) and for native GCN4-p1 (observed: $4037.7 \mathrm{Da}$; calculated $\left(\mathrm{C}_{176} \mathrm{H}_{297} \mathrm{~N}_{52} \mathrm{O}_{54} \mathrm{~S}\right)$ : 4037.7 Da). The purity by HPLC was $>95 \%$. Peptide concentration was determined in triplicate under denaturing conditions in $6 \mathrm{M} \mathrm{GdnHCl}(\mathrm{pH} \mathrm{6.5)}$ by monitoring the tyrosine absorbance at $275 \mathrm{~nm}$, using an extinction coefficient ${ }^{23}$ of $1470 \mathrm{M}^{-1} \mathrm{~cm}^{-1}$.

\section{Cross-linking $(i, i+7)$ Cys GCN4-p1}

Intramolecular cross-linking of Cys residues by 1 was performed. It was found necessary to perform the cross-linking reaction in the presence of guanidinium hydrochloride to disrupt the dimeric coiled coil interaction of $(i, i+7)$ Cys GCN4-p1. In a total volume of $500 \mu \mathrm{L}, 47 \mathrm{mM}$ Tris-HCl buffer (pH 8.0), uncross-linked $(i, i+7)$ Cys GCN4-p1 $(0.75 \mathrm{mM})$, TCEP $(0.83 \mathrm{mM})$ and GdnHCl $(2.3 \mathrm{M})$ were combined and incubated for $18 \mathrm{~h}$ at room temperature, under nitrogen to ensure the cysteine residues were in their reduced state. To the aqueous solution, $500 \mu \mathrm{L}$ DMSO containing $0.6 \mu \mathrm{mol}$ of 1 was added, giving a reagent concentration of $0.6 \mathrm{mM}$. This solution was stirred for 20 min protected from light in a $40^{\circ} \mathrm{C}$ waterbath, followed by the addition of $60 \mu \mathrm{L}$ of a $10 \mathrm{mM}$ solution of 1 . The reaction mixture was stirred in a $40^{\circ} \mathrm{C}$ waterbath for a further 20 min protected from light followed by 20 min exposed to light. The solvent was removed by high vacuum pump and the peptide was purified by HPLC (Zorbax SB-C18 column) using a linear gradient from 0 $75 \%$ acetonitrile $/ \mathrm{H}_{2} \mathrm{O}(+0.1 \%$ trifluoroacetic acid $)$ over the course of $50 \mathrm{~min}$ for $(i, i+7)$ Cys GCN4-p1-X (eluted at $47.4 \%$ acetonitrile). The peptide primary structure was confirmed by MALDI-MS: $(i, i+7)$ Cys GCN4-p1-X (observed $4377.05 \mathrm{Da}$; calculated $\left(\mathrm{C}_{194} \mathrm{H}_{314} \mathrm{~N}_{55} \mathrm{O}_{54 \mathrm{~S}} \mathrm{~S}_{3}\right)$ : $4377.09 \mathrm{Da}$ ). Concentration of the dark-adapted cross-linked peptide was determined by monitoring the azobenzene crosslinker absorbance at $367 \mathrm{~nm}$, using a molecular extinction coefficient ${ }^{8}$ of $28000 \mathrm{M}^{-1} \mathrm{~cm}^{-1}$.

\section{Circular dichroism measurements}

Circular dichroism measurements were performed using a Jasco Model J-710 spectropolarimeter. All measurements were carried out in thermostatted quartz cuvettes $(0.1 \mathrm{~cm}$ or $1.0 \mathrm{~cm}$ pathlength $)$. Temperatures were measured using a microprobe placed directly into the sample cell. All samples were dissolved in $5 \mathrm{mM}$ acetate buffer $(\mathrm{pH} 5)$ or $5 \mathrm{mM}$ phosphate buffer $(\mathrm{pH} 7)$. DTT ( $3 \mathrm{mM})$ was present in the uncross-linked peptide samples to ensure that cysteine residues were in the reduced form. Spectra reported are averages of three individual experiments of five scans each, with the appropriate background spectrum subtracted. A scan speed of $10 \mathrm{~nm} \mathrm{~min}{ }^{-1}$, with a $0.5 \mathrm{~nm}$ bandwidth and a $4 \mathrm{~s}$ response time was used. All $\mathrm{CD}$ signals are reported using mean residue ellipticity. The mean residue weight used for native GCN4-p1 was 122.4 and for $(i, i+7)$ Cys GCN4-p1 in the uncross-linked and cross-linked form was 123.7.

Guanidinium hydrochloride $(\mathrm{GdnHCl})$ denaturation curves were measured by monitoring the mean residue ellipticity at $222 \mathrm{~nm}\left(\theta_{222} \mathrm{~nm}\right)$ as a function of $\mathrm{GdnHCl}$ concentration. Thermal denaturation studies were performed by monitoring $\theta_{222} \mathrm{~nm}$ at various temperatures ranging from $10^{\circ} \mathrm{C}$ to $70^{\circ} \mathrm{C}$ in $5^{\circ} \mathrm{C}$ increments. The samples were allowed to equilibrate at each temperature for 5 min prior to data acquisition.

\section{UVIVIS spectroscopy and photo-isomerisation}

UV/VIS absorbance spectra were measured with a Perkin-Elmer Lambda 2 spectrophotometer using the same thermostatted quartz cuvettes $(0.1 \mathrm{~cm}$ or $1.0 \mathrm{~cm}$ pathlength) in which the CD data were collected. To obtain rates of thermal isomerisation, the dependence of the absorbance at 367 $\mathrm{nm}$ was recorded with respect to time. The resulting curve was fitted to an exponential decay expression using the program IGOR (Wavemetrics).

Photo-isomerisation was accomplished by irradiating thermostatted peptide solutions with a 70 W metal halide Tri-Lite Lamp (World Precision Instruments Inc, FL) coupled to a $370 \mathrm{~nm} \pm 10 \mathrm{~nm}$ band pass filter (Harvard Apparatus Canada, PQ). Photo-isomerisation was complete (as judged by the lack of any further changes in UV spectra) in $\leqslant 10 \mathrm{~min}$. A limitation of the azobenzene chromophore is that it is not possible to achieve a complete isomerisation of a sample to the cis form. ${ }^{8,9}$ The proportion of the sample converted to cis upon irradiation of cross-linked peptide was determined by fitting its UV/VIS spectra with those of pure trans and cis samples obtained by 
HPLC separation ${ }^{9}$ of a monomeric peptide containing the same azobenzene moiety. Given a measurement of $p_{c i s}$ (the proportion of a sample converted to cis) it is possible to estimate the CD spectrum of a sample with $100 \%$ conversion to cis:

$$
\theta_{\lambda}{ }^{100 \% \text { cis }}=\left\{\theta_{\lambda}{ }^{\text {irradiated }}-\left(1-p_{\text {cis }}\right) \theta_{\lambda}{ }^{\text {dark }}\right\} / p_{\text {cis }}
$$

where $\theta_{\lambda}{ }^{\text {irradiated }}$ is the $\mathrm{CD}$ signal at a wavelength of $\lambda \mathrm{nm}$ after irradiation and $\theta_{\lambda}{ }^{\text {dark }}$ is the corresponding signal for the dark-adapted peptide. This extrapolation makes the assumption that the observed CD spectrum is a linear combination of the CD spectra of pure cis and pure trans peptides. The possibility that a coiled coil formed from one cis and one trans peptide gives a CD spectrum different from the spectrum of an equimolar mixture of cis-cis and trans-trans peptides is neglected. Nevertheless, the expression provides a useful estimate of the helicity for a sample with complete conversion of the cross-linker to the cis conformation.

\section{Results}

\section{Peptide design}

The starting point for the design of a GCN4-p1 derivative incorporating the azobenzene-based cross-linker 1 was the high-resolution X-ray crystal structure of O'Shea et al. ${ }^{13}$ It was decided to incorporate the cysteine residues to be cross-linked at a relative spacing of $i$ to $i+7$ as the utility of this approach had already been demonstrated for a 16 -residue monomeric peptide. ${ }^{8}$ In addition it was decided that the position $i+3$, occupying a position under the cross-linker, should be set to a $\beta$-branched amino acid such as isoleucine. This idea had its origin in preliminary modelling studies that indicated that such a bulky residue would increase the degree of photo-control by participating in steric interactions with the trans form of the linker. ${ }^{8}$ Subsequent experimental work and improved modelling has shown this idea to be in error. In fact, the residue types below the crosslinker have little effect on the degree of photo-control. ${ }^{9 a}$

Once the decision had been made to mutate three residues to cysteine, isoleucine and cysteine with a relative spacing of $(i, i+3, i+7)$ the next step was to determine where in the sequence to introduce these changes. Consideration of the sequence variability of coiled coils ${ }^{15}$ complemented by a sequence alignment of GCN4-p1 with homologous protein sequences from the SWISS-PROT databank, ${ }^{24}$ indicated that the $\mathrm{b}, \mathrm{c}$ and $\mathrm{f}$ positions from the heptad repeat are most tolerant to alteration. It was decided that the mutation to cysteine of two consecutive f heptad-repeat positions was advantageous and produced the required relative inter-cysteine spacing of $i$ to $i+7$. This would ensure that the cysteine/cross-linker/cysteine moiety would lie on the solvent exposed side of the coiled coil (Fig. 2B). Thus the $(i+3)$ residue, which is to be mutated to isoleucine, would lie in the b heptad position and as such would also be amenable to change (Fig. 2B). Given this decision the next question was where in the sequence of GCN4-p1 (Fig. 2A) to introduce the changes. The only places where the desired changes could be made without altering the charge of the peptide were S14C, Y17I, N21C (Fig. 2A). Substitution of residue Tyr17 removes a useful means of assessing the concentration of the peptide spectroscopically. ${ }^{14}$ As a consequence of this a further modification A24Y was included, thereby reintroducing a tyrosine residue (in the b position of the next heptad repeat).

The final stage of the design involved checking whether the modifications outlined above would result in obvious structural problems. The amino acid modifications were incorporated into the high-resolution X-ray crystal structure ${ }^{13}$ of GCN4-p1 (Fig. 3A) using the SYBYL package. Examination with molecular graphics showed that no obvious conflict was produced by the changes. To assess the compatibility of the cross-linked structure with coiled coil formation, two models were produced: one with the azobenzene group in a cis conformation and the other with the azobenzene in the trans form. The conformation of each form of the azobenzene cross-linker was the result of ab initio geometry optimisation using the $6-31 \mathrm{G}^{*}$ basis function with Gaussian 98 . The GCN4-p1 dimer structure was then separated into two monomers. For each of the monomers the cross-linker was joined to the cysteine side chains. Each cross-linked monomer was then subjected to energy minimisation with the AMBER4.1 force field, ${ }^{20}$ while the cross-linker was constrained to its $a b$ initio low-energy conformation. After energy minimisation the two monomers modelled with the cross-linker in a cis conformation, remained fully helical and could be rejoined to form a dimer 

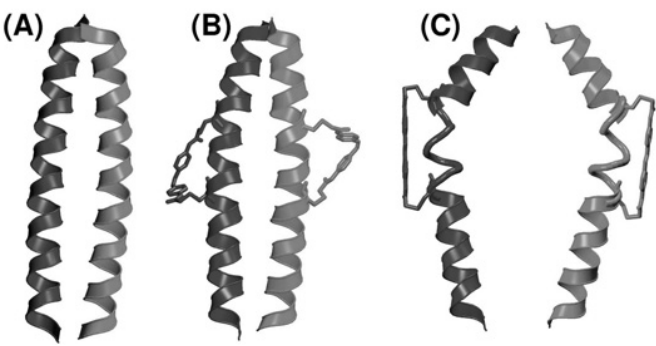

Fig. 3 Molecular models of the proposed cross-linked GCN4-p1. A comparison between the structures of (A) the X-ray crystal structure ${ }^{13}$ for GCN4-p1, (B) a model for $(i, i+7)$ Cys GCN4-p1X with both cross-linkers in a cis conformation and (C) a model for $(i, i+7)$ Cys GCN4-p1X with both cross-linkers in a trans conformation. In all cases $\alpha$-helical sections of the structure are marked with ribbons and areas where helicity is disrupted are marked by a tube, an atomic representation is used for both cysteine residues and the cross-linker. Picture produced using $\mathrm{VMD}^{21}$ and Raster $3 \mathrm{D}^{22}$

(Fig. 3B). In contrast, the trans conformation of the cross-linker forced a considerable bend in the helix (Fig. 3C) that was expected to substantially destabilise coiled coil dimer formation.

\section{Characterisation of uncross-linked $(i, i+7)$ Cys GCN4-p1 in comparison to native GCN4-p1}

After synthesis and purification of the peptide the conformation of uncross-linked $(i, i+7)$ Cys GCN4-p1 was analysed by CD spectropolarimetry (Fig. 4). All experiments were conducted in the presence of DTT to ensure that the cysteine side chains were in a reduced form. The concentration of the peptide was varied in the range $6 \mu \mathrm{M}$ to $50 \mu \mathrm{M}$ (Table 1). Due to problems with solubility in the cross-linked form of $(i, i+7)$ Cys GCN4-p1 (see below), the majority of CD analysis was performed at $\mathrm{pH}$ 5. For comparison with previously published studies, the un-crosslinked $(i, i+7)$ Cys GCN4-p1 was also analysed at $\mathrm{pH} 7$. As a control, native GCN4-p1 was synthesised by solid-phase peptide synthesis and analysed at $\mathrm{pH} 5$ and $\mathrm{pH} 7$ to allow comparison with previously published results (Table 2).

$\mathrm{CD}$ analysis of native GCN4-p1 at $10.5^{\circ} \mathrm{C}$ gave $\theta_{222} \mathrm{~nm}$ of -28970 degrees $\mathrm{cm}^{2} \mathrm{dmol}^{-1}(50 \mu \mathrm{M}$, $\mathrm{pH} 7$ ) which compares to -33200 degrees $\mathrm{cm}^{2} \mathrm{dmol}^{-1}\left(35 \mu \mathrm{M}, 0{ }^{\circ} \mathrm{C}, \mathrm{pH}\right.$ 7) reported by Lumb and co-workers. ${ }^{25}$ The native GCN4-p1 was also analysed at pH $5\left(5 \mu \mathrm{M}, 10.5^{\circ} \mathrm{C}\right)$ and gave $\theta_{222 \mathrm{~nm}}$ of -21670 degrees $\mathrm{cm}^{2} \mathrm{dmol}^{-1}$ which compares well with a reported $\theta_{222 \mathrm{~nm}}$ value of -22500 degrees $\mathrm{cm}^{2} \mathrm{dmol}^{-1}\left(12-15 \mu \mathrm{M}, 10^{\circ} \mathrm{C}, \mathrm{pH}\right.$ 5.5) for a GCN4-p1 Ser14Ala mutant in which no change in stability was observed from the native peptide at $\mathrm{pH} 5{ }^{26,27}$ These control experiments demonstrated that the conditions and techniques used here are not inconsistent with work reported by other groups.

Comparison between the values in Tables 1 and 2 shows that the amino acid mutations introduced into $(i, i+7)$ Cys GCN4-p1 have resulted in a substantial destabilisation of coil-coiled formation compared to native GCN4-p1. This is true at both $\mathrm{pH} 7$ and $\mathrm{pH}$ 5. It should be noted, however, that the marked concentration dependence of $\theta_{222} \mathrm{~nm}$ indicates that some peptide association is occurring. A similar concentration dependence is also observed in thermal denaturation curves (Fig. 4 inset). It should be noted that melting occurs at temperatures around $40^{\circ} \mathrm{C}$ lower than native GCN4-p1. ${ }^{14}$

To test whether this unexpected destabilisation was a result of an alteration of the intrinsic helicity of the peptide or the disruption of the coiled coil interface, the effect of the solvent trifluorethanol (TFE) was assessed. This solvent promotes the formation of $\alpha$-helices in monomeric peptides ${ }^{28}$ producing a marked increase in the size of the 209 and $222 \mathrm{~nm}$ minima in CD spectra. Its effect on coiled coil peptides is more complex..$^{29,30}$ The presence of $50 \%$ TFE causes the breakdown of dimeric coiled coils into monomers. ${ }^{30}$ This effect is accompanied by changes to the CD spectrum: a slight decrease in the magnitude of the $222 \mathrm{~nm}$ minimum and an increase by a similar amount in the size of the minimum at $209 \mathrm{~nm}^{29}$

For uncross-linked $(i, i+7)$ Cys GCN4-p1 the addition of 50\% TFE produced a marked increase in the size of the 209 and $222 \mathrm{~nm}$ minima in the CD spectra (Fig. 4B). The $\theta_{222} \mathrm{~nm}$ signal showed 

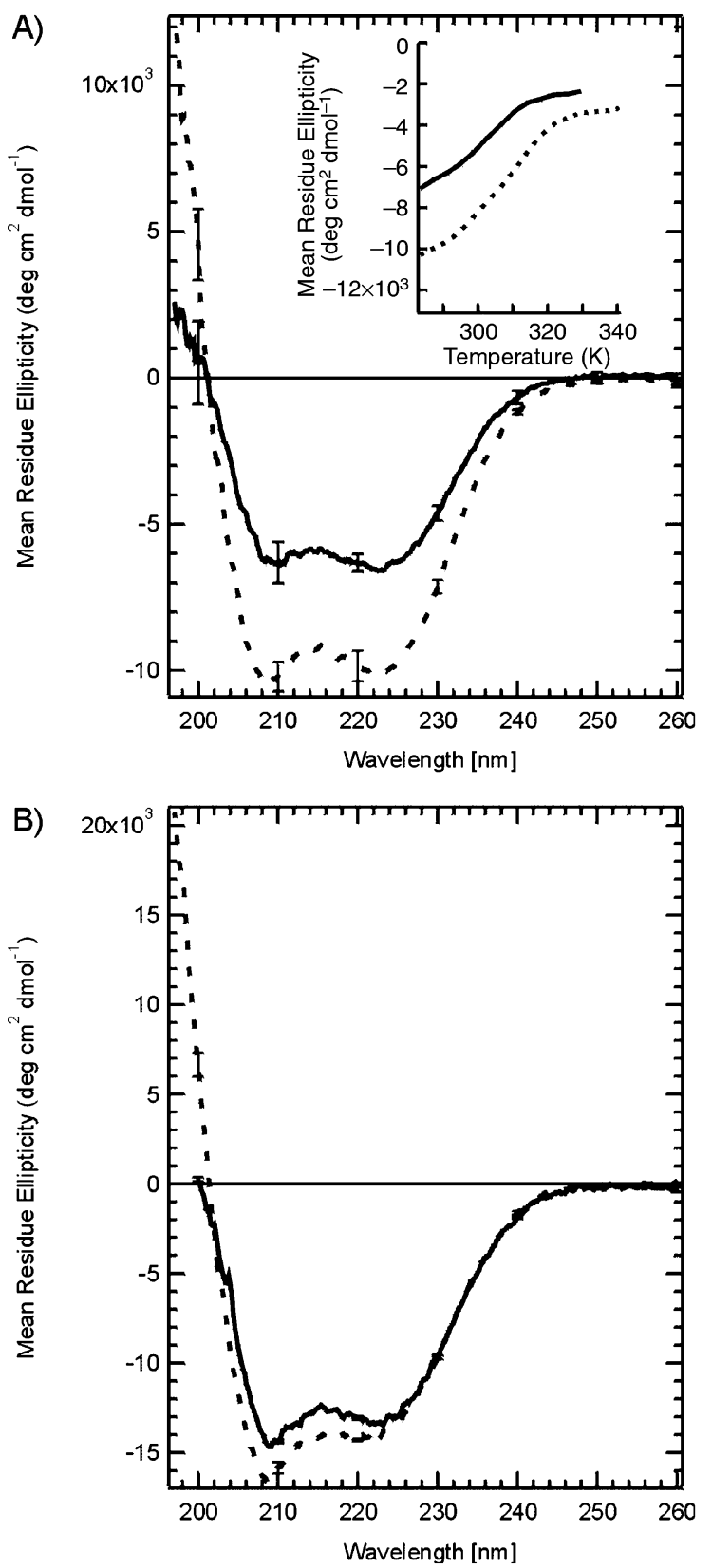

Fig. 4 Secondary structure of un-crosslinked $(i, i+7)$ Cys GCN4-p1. (A) CD spectra of $6 \mu \mathrm{M}$ (solid line) and $50 \mu \mathrm{M}$ (dashed line) un-crosslinked $(i, i+7)$ Cys GCN4-p1 (5 mM sodium acetate buffer pH 5.0, 3 mM DTT, $10^{\circ} \mathrm{C}$ ). (Inset) thermal denaturation of $6 \mu \mathrm{M}$ (solid line) and $50 \mu \mathrm{M}$ (dashed line) un-crosslinked $(i, i+7) \mathrm{Cys}$ GCN4-p1. (B) CD spectra of $6 \mu \mathrm{M}$ (solid line) and $50 \mu \mathrm{M}$ (dashed line) un-crosslinked $(i, i+7)$ Cys GCN4-p1 in the presence of $50 \%$ TFE $/ 50 \% 5 \mathrm{mM}$ sodium acetate buffer $\mathrm{pH} 5.0,3 \mathrm{mM}$ DTT, $10^{\circ} \mathrm{C}$.

only a slight dependence on the concentration of the peptide (Table 1), consistent with the formation of a monomer. Furthermore, the minimum at $209 \mathrm{~nm}$ falls to a greater extent than that at $222 \mathrm{~nm}$. Taken together these observations suggest that 50\% TFE breaks down a partially formed coiled coil present in a purely aqueous environment. Even in 50\% TFE the peptide is of only limited 
Table 1 Analysis of un-crosslinked $(i, i+7)$ Cys GCN4-p1 by circular dichroism spectropolarimetry ${ }^{a}$

\begin{tabular}{lrr}
\hline & \multicolumn{1}{l}{$\theta_{222 \mathrm{~nm}}$} \\
\cline { 2 - 3 }$(i, i+7)$ Cys GCN4-p1 concentration $/ \mu \mathrm{M}$ & $\mathrm{pH} 5$ & $\mathrm{pH} \mathrm{7}$ \\
\hline 6 & $-6520 \pm 500$ & $-7030 \pm 700$ \\
12 & $-8600 \pm 100$ & $-9770 \pm 80$ \\
25 & $-10010 \pm 200$ & \\
50 & $-10020 \pm 500$ & $-10690 \pm 900$ \\
6 with $50 \%$ TFE & $-13330 \pm 200$ & \\
50 with $50 \%$ TFE & $-13940 \pm 200$ & $-14290 \pm 600$
\end{tabular}

${ }^{a}$ All CD measurements were performed at $10 \pm 1^{\circ} \mathrm{C}$ in $5 \mathrm{mM} \mathrm{NaOAc}(\mathrm{pH}$ 5.0) or $5 \mathrm{mM} \mathrm{NaPO}_{4}(\mathrm{pH} 7.0)$ with $3 \mathrm{mM}$ DTT. Mean residue ellipticity $\left(\theta_{222 \mathrm{~nm}}\right)$ is reported in degrees $\mathrm{cm}^{2} \mathrm{dmol}^{-1}$. The average of three individual experiments is quoted, followed by the standard deviation.

Table 2 Analysis of native GCN4-p1 by circular dichroism spectropolarimetry $^{a}$

\begin{tabular}{ll}
\hline GCN4-p1 concentration $/ \mu \mathrm{M}$ & $\theta_{222 \mathrm{~nm}}$ \\
\hline 5 (pH 5) & $-21670 \pm 100$ \\
50 (pH 7) & $-28970 \pm 200$ \\
50 (with 50\% TFE pH 7) & $-30860 \pm 200$
\end{tabular}

${ }^{a}$ All CD measurements were performed at $10 \pm 1^{\circ} \mathrm{C}$ in $5 \mathrm{mM} \mathrm{NaOAc}(\mathrm{pH} 5.0)$ or $5 \mathrm{mM} \mathrm{NaPO}_{4}(\mathrm{pH} 7.0)$ with $3 \mathrm{mM}$ DTT. Mean residue ellipticity $\left(\theta_{222 \mathrm{~nm}}\right)$ is reported in degrees $\mathrm{cm}^{2} \mathrm{dmol}^{-1}$. The average of three individual experiments is quoted, followed by the standard deviation.

helicity (comparing values in Tables 1 and 2). This shows that the amino acid changes made affected the helix propensity of the peptide both in the monomeric and associated (presumably coiled coil) forms.

\section{Characterisation of cross-linked $(i, i+7)$ Cys GCN4-p1}

The $(i, i+7)$ Cys GCN4-p1 peptide was cross-linked as described above and is denoted here as $(i$, $i+7)$ Cys GCN4-p1X. The conformation of $(i, i+7)$ Cys GCN4-p1X was assessed using CD analysis both for the dark-adapted state and following irradiation. Initial results from experiments conducted at $\mathrm{pH} 7.0$ presented a complication. The peptide was soluble in the dark and immediately after irradiation. However, if the sample was left following irradiation a precipitate formed over a number of hours. This precipitate formed at peptide concentrations as low as $3 \mu \mathrm{M}$. This complication was alleviated by altering the experimental conditions. At $\mathrm{pH} 5.0$ the precipitation was not observed for irradiated samples with concentrations up to $12 \mu \mathrm{M}$. At this lower $\mathrm{pH}$ the trans form of the cross-linked peptide showed no formation of precipitate at concentrations up to $50 \mu \mathrm{M}$ (Table 3). Analysis of a GCN4-p1 system at $\mathrm{pH} 5$ has been reported previously by Sosnick and co-workers. ${ }^{27}$ Although we are currently unable to explain this phenomenon, there is a histidine residue (residue number 18) under the cross-linker (in the $i+4$ position relative to cysteine 14) that might lead to the $\mathrm{pH}$ dependent tendency of the peptide to precipitate.

The CD analysis for the cross-linked peptide is illustrated in Fig. 5 and the corresponding $\theta_{222} \mathrm{~nm}$ values are reported in Table 3. A fit of the UV/VIS absorption spectrum (Fig. 5A) with "pure" cis 
Table 3 Analysis of $(i, i+7)$ Cys GCN4-p1X by circular dichroism spectropolarimetry $^{a}$

\begin{tabular}{|c|c|c|c|}
\hline \multirow{2}{*}{\multicolumn{2}{|c|}{$(i, i+7)$ Cys GCN4-p1X concentration $/ \mu \mathrm{M}$}} & \multicolumn{2}{|l|}{$\theta_{222 \mathrm{~nm}}$} \\
\hline & & $\mathrm{pH} 5$ & $\mathrm{pH} 7^{b}$ \\
\hline \multirow[t]{3}{*}{5} & Dark adapted & $-13020 \pm 370$ & $-10550 \pm 500$ \\
\hline & Irradiated $^{c}$ & $-16330 \pm 640$ & $-13340 \pm 200$ \\
\hline & Estimated $100 \%$ cis $^{d}$ & -18350 & \\
\hline \multirow[t]{3}{*}{12} & Dark adapted & -16590 & \\
\hline & Irradiated $^{c}$ & -18800 & \\
\hline & Estimated $100 \%$ cis $^{d}$ & -20200 & \\
\hline \multirow[t]{3}{*}{50} & Dark adapted & $-16310 \pm 330$ & \\
\hline & Irradiated $^{c}$ & $-18990 \pm 160$ & \\
\hline & Estimated $100 \%$ cis $^{d}$ & -20600 & \\
\hline
\end{tabular}

a All CD measurements were performed at $10 \pm 1^{\circ} \mathrm{C}$ in $5 \mathrm{mM} \mathrm{NaOAc}(\mathrm{pH} 5.0)$ or $5 \mathrm{mM} \mathrm{NaPO}_{4}(\mathrm{pH} \mathrm{7.0})$. Mean residue ellipticity $\left(\theta_{222 \mathrm{~nm}}\right)$ is reported in degrees $\mathrm{cm}^{2}$ $\mathrm{dmol}^{-1}$. The average of three individual experiments is quoted, followed by standard deviation. ${ }^{b}$ Upon thermal relaxation of $(i, i+7)$ Cys GCN4-p1X at $\mathrm{pH} 7$, precipitate was formed, therefore was not fully reversible. ${ }^{c}$ After exposure to $370 \pm 10 \mathrm{~nm}$ light for $10 \mathrm{~min}\left(70 \mathrm{~W}\right.$ source). ${ }^{d}$ Estimated value for a peptide sample where all the cross-linker is in the cis conformation. Estimate derived from dark and irradiated values and a measurement that the irradiated sample represents a mixture of $62 \%$ cis and $38 \%$ trans (see text).

and trans spectra showed that the irradiated sample was composed of a mixture of $62 \%$ cis and $38 \%$ trans azobenzene chromophore (see Methods). Taking this into account it is possible to estimate both the CD spectrum (Fig. 5B) and $\theta_{222} \mathrm{~nm}$ for a sample composed of only the cis cross-linker conformation. All spectra are characteristic of a partially formed $\alpha$-helix. Furthermore, the increase in the magnitude of the $\theta_{222} \mathrm{~nm}$ minimum with concentration shows that the degree of helicity is concentration dependent (Table 3). The irradiated and corrected "pure" cis spectra clearly show the features consistent with coiled coil formation: ${ }^{14,29}$ namely that the $222 \mathrm{~nm}$ minimum is lower than that at $209 \mathrm{~nm}$ (Fig. 5B).

There is a clear difference in the signal size with more helicity in the illuminated and "pure" cis spectra than that for the dark-adapted peptide (Table 3). Furthermore the $222 \mathrm{~nm}$ minimum is lower than $209 \mathrm{~nm}$ minimum and this tendency increases as the signal magnitude is increased. A similar effect has been observed for native GCN4-p1 ${ }^{14}$ Comparison of the magnitude of the $\theta_{222 \mathrm{~nm}}$ signal for $(i, i+7)$ Cys GCN4-p1X at a concentration of $5 \mu \mathrm{M}$ (Table 3 ) with that for the native GCN4-p1 (Table 2) is instructive. Expressed as a proportion of the value for the native peptide the $\theta_{222} \mathrm{~nm}$ signal for the dark-adapted state is $60 \%$ and upon illumination this increases to $75 \%$. Taking into account the correction to estimate a "pure" cis the signal increases to $85 \%$. This demonstrates that the cis conformation of the cross-linker is helix stabilising and therefore able to restore a large proportion of the coiled coil formation disrupted by the amino acid modifications incorporated in $(i, i+7)$ Cys GCN4-p1. In contrast the helicity, and consequently coiled coil formation, of the dark-adapted state is considerably lower than that for the "pure" cis showing that a degree of photo-control has been achieved. The greater concentration dependence found for the $\theta_{222 \mathrm{~nm}}$ signal for the dark-adapted case compared to illuminated or pure cis (Table 3 ) is consistent with decreased coiled coil stability.

An unexpected observation was that the trans (dark-adapted) conformation effects helix stabilisation (Table 3) in comparison to the uncross-linked peptide (Table 1). When expressed as a proportion of the value for the native peptide the $\theta_{222 \mathrm{~nm}}$ signal for the dark-adapted state, at $5 \mu \mathrm{M}$, is $60 \%$. In comparison the uncross-linked peptide at $6 \mu \mathrm{M}$ exhibited only $30 \%$ of the native $\theta_{222} \mathrm{~nm}$ signal. In previous studies of monomeric peptides using the cross-linker with an $(i, i+7)$ or an $(i$, $i+4)$ inter-cysteine spacing, ${ }^{8,9}$ we have observed that the trans form of the linker destabilises the structure compared to the uncross-linked form of the peptide. Increased stability in the trans form 

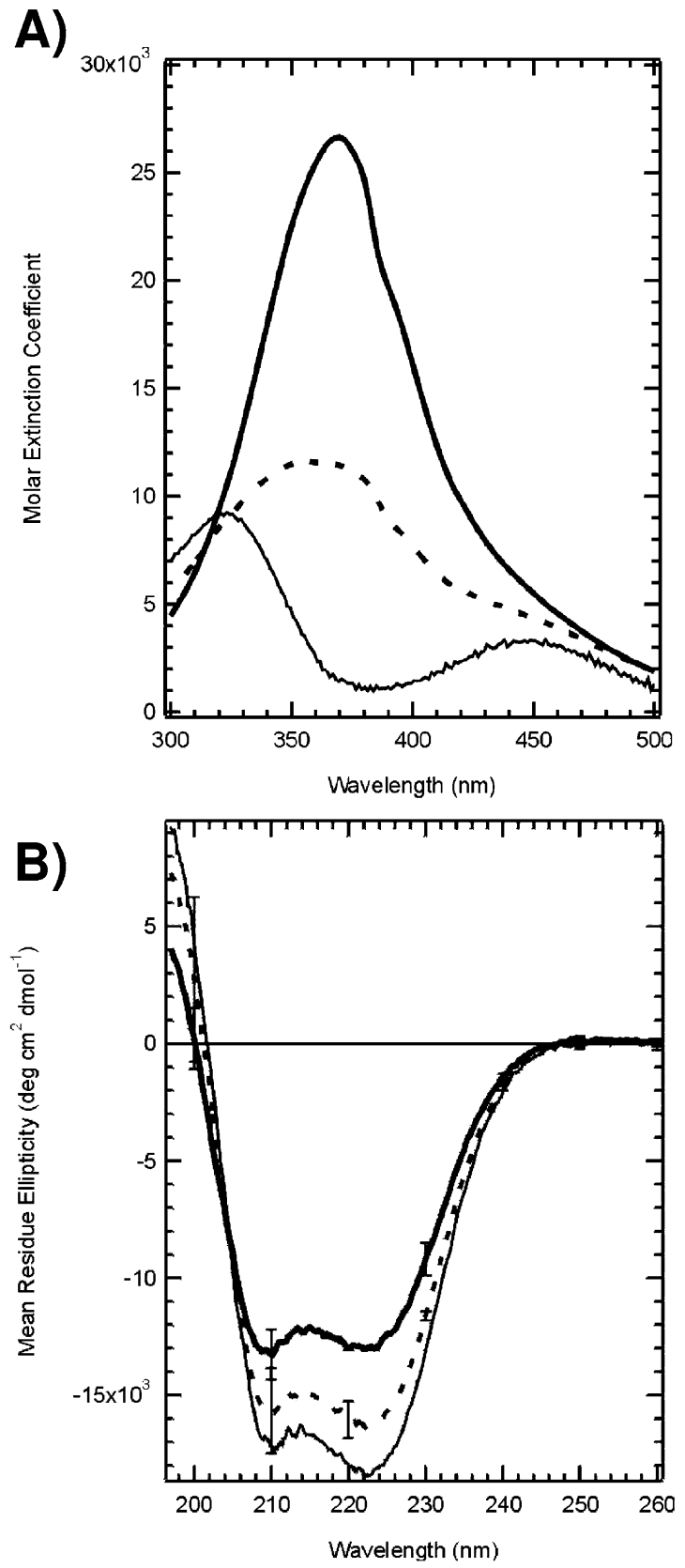

Fig. 5 Effects of photo-irradiation on $(i, i+7)$ Cys GCN4-p1X. (A) UV Spectra of $(i, i+7)$ Cys GCN4-p1X, dark-adapted (solid line), irradiated (dashed line) and 100\% cis (thin solid line). (B) CD spectra of $6 \mu \mathrm{M}$ darkadapted $(i, i+7)$ Cys GCN4-p1X (thick solid line) and irradiated $(i, i+7)$ Cys GCN4-p1X (dashed line). Conditions $5 \mathrm{mM}$ sodium acetate buffer, $\mathrm{pH} 5.0,10^{\circ} \mathrm{C}$; irradiation used $370 \mathrm{~nm}$ light for $10 \mathrm{~min}, 70 \mathrm{~W}$ source. Calculated $100 \%$ cis $\mathrm{CD}$ spectrum represented by thin solid line. 


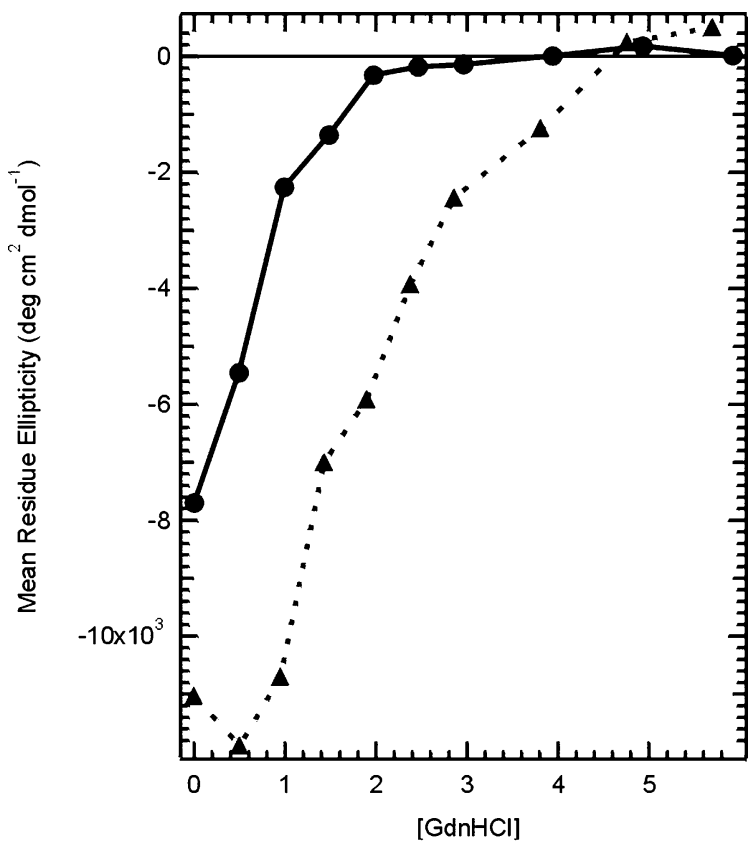

Fig. 6 Chemical denaturation of uncross-linked and cross-linked $(i, i+7)$ Cys GCN4-p1. Effect of increasing $\mathrm{GdnHCl}$ concentration on $\mathrm{CD}$ signal of $6 \mu \mathrm{M}$ uncross-linked $(i, i+7) \mathrm{Cys}$ GCN4-p1 (solid line, circles) and 6 $\mu \mathrm{M}$ dark-adapted (trans) cross-linked $(i, i+7)$ Cys GCN4-p1 (dotted line, triangles) (5 mM sodium phosphate buffer, $\mathrm{pH} 7,3 \mathrm{mM}$ DTT, $\left.10^{\circ} \mathrm{C}\right)$.

was further confirmed by performing $\mathrm{GdnHCl}$ denaturation experiments on both the uncrosslinked $(i, i+7)$ Cys GCN4-p1 and the (trans) cross-linked $(i, i+7)$ Cys GCN4-p1X at the same concentration (Fig. 6). From this analysis it was apparent that the cross-linked form behaves differently from the uncross-linked system in that it is more stable to chemical denaturation by $\mathrm{GdnHCl}$.

A point of interest is the influence that the environment has on the photo-isomerisation properties of the azobenzene-based cross-linker. As discussed above, it was found that in this case the photo-stationary state on illumination with $370 \mathrm{~nm}$ light was $62 \%$ cis $/ 38 \%$ trans. This can be compared to results obtained for monomeric peptides where the limiting percentage of molecules in the cis conformation was $77 \%$ to $90 \%{ }^{9}$ It was also noted that a longer irradiation time was necessary to reach a photo-stationary state (unpublished results). Table 4 shows the thermal relaxation half-lives for the cis conformation to revert to a trans conformation at two different temperatures. The half-times obtained at these temperatures were similar to those previously reported for the original monomeric peptide. ${ }^{8}$ Therefore the behaviour of the cross-linker upon relaxation found here is similar to other applications, despite distinct differences in the properties of the photo-isomerisation process.

Table 4 Temperature dependence of thermal relaxation of the cis cross-linked peptides in the dark

\begin{tabular}{lll}
\hline Temperature $/{ }^{\circ} \mathrm{C}^{a}$ & $\mathrm{JRK}\{$ ValAib $\}-\mathrm{X}^{b} \tau_{\frac{1}{2}} / \min$ & $(i, i+7) \mathrm{Cys}$ GCN4-p1X $\tau_{\frac{1}{2}} / \mathrm{min}$ \\
\hline 25 & 22 & 20 \\
31 & 7.8 & 10 \\
${ }^{a}$ Temperatures are accurate to $\pm 1{ }^{\circ} \mathrm{C} .{ }^{b}$ Values from ref. 8. \\
\hline
\end{tabular}




\section{Discussion}

The results obtained are encouraging. We set out to produce a coiled coil peptide whose helicity, and as a consequence dimerisation, is under photo-control. Fig. 5 shows clear evidence that this has been achieved. This success is tempered by the fact that the mutations introduced to allow crosslinking caused a radical disruption of the coiled coil prior to the cross-linking step. The addition of the cross-linker restored a more native conformation with the exception of the need to avoid neutral $\mathrm{pH}$ to avoid precipitation.

Could such a disruption have been expected? It is instructive to make a comparison with results obtained by Zitzewitz and co-authors. ${ }^{31}$ These authors produced a series of modified GCN4-p1 peptides in which all four of the external heptad repeat $\mathrm{f}$ positions: Asp7, Ser14, Asn21 and Lys28 (see Fig. 2A) were mutated. ${ }^{31}$ The peptides produced were designated by the mutations made at the f positions: f-DSNK is the native wild type sequence whereas f-AAAA refers to a mutant where all four $\mathrm{f}$ position residues are mutated to alanine. ${ }^{31}$ It was found ${ }^{31}$ that the experimental differences in the free energy of coiled coil formation could be reasonably reproduced using a simple consideration of the helix propensity free energy scale developed by O'Neil and DeGrado. ${ }^{32}$ This scale was itself derived by determining the effect on the stability of modifications at the heptad repeat $\mathrm{f}$ position of another coiled coil peptide, ${ }^{32}$ and so is particularly applicable to this situation.

Applying a similar consideration to that applied by Zitzewitz and co-authors ${ }^{31}$ we can estimate the effect of the mutations introduced into $(i, i+7)$ Cys GCN4-p1 (Fig. 2). The effect expected from the individual mutations on the free energy of coiled coil are as follows $\mathrm{S} 14 \mathrm{C}:+0.24 \mathrm{kcal} \mathrm{mol}^{-1}$, Y17I: $-0.12 \mathrm{kcal} \mathrm{mol}^{-1}$, N21C: $-0.32 \mathrm{kcal} \mathrm{mol}^{-1}, \mathrm{~A} 24 \mathrm{Y}+1.20 \mathrm{kcal} \mathrm{mol}^{-1}$. Overall, we could expect that the four mutations would result in the destabilisation of the coiled coil by $1.0 \mathrm{kcal}$ $\mathrm{mol}^{-1}$ (compared to a free energy of folding for the native coiled coil of $-10.6 \mathrm{kcal} \mathrm{mol}^{-131}$ ). This can be compared to the estimates produced by this method for reductions in the folding free energy produced by mutations f-NNNN and f-GGGG of 1.88 and $2.44 \mathrm{kcal} \mathrm{mol}^{-1}$ respectively. These estimates are in reasonable agreement with the experimental values for differences found. ${ }^{31}$ Comparing the values shows that we could expect that the mutations introduced into $(i, i+7)$ Cys GCN4-p1 would have a smaller destabilising effect than f-NNNN and considerably lower than that found for f-GGGG. Experimentally the f-NNNN peptide is $90 \%$ folded at a peptide concentration of $10 \mu \mathrm{M}$ while $\mathrm{f}-\mathrm{GGGG}$ is $\sim 70 \%$ folded at a $100 \mu \mathrm{M}$ concentration. ${ }^{31}$ From these considerations it is not unreasonable to expect that $(i, i+7)$ Cys GCN4-p1 would be at least $90 \%$ folded at concentrations above $10 \mu \mathrm{M}$. However, comparison between values for $\mathrm{pH} 7$ from Tables 1 and 2 shows that less than $35 \%$ of the peptide is folded at a concentration of $12 \mu \mathrm{M}$. The deviation between expected and observed effects is dramatic.

It is clear that the destabilisation of $(i, i+7)$ Cys GCN4-p1 before cross-linking cannot be accounted for by a simple consideration of the effects on the helix propensity. What other factor could account for the phenomenon? Coiled coil peptides have been reported to accommodate cysteine residues without large disruption of their structure. ${ }^{32-34}$ The mutations introduced (S14C, Y17I, N21C and A24Y) involve the substitution of reasonably hydrophilic amino acids with larger hydrophobic residues. It should be noted that cysteine is a hydrophobic amino acid, occurring next to alanine in a scale of hydrophobicity. ${ }^{35}$ Consideration of the helical wheel diagram for a coiled coil (Fig. 2B), shows that the mutations are introduced in the $b$ and $f$ positions of consecutive heptad repeats. In addition the residue Leu13 lies in a solvent exposed position $^{13}$ (heptad repeat e) next to the mutations. This, in effect, creates a stripe of hydrophobic amino acids on what should be the external face of the coiled coil (Fig. 7). The major force in the assembly of a coiled coil is the association of the hydrophobic surfaces at the centre of the coil opposite this external face. It can be imagined that interactions between the hydrophobic residues introduced by the mutations and partly formed coiled coils could interfere with normal assembly and may be involved in the precipitation. Analysis of a model for the uncross-linked mutant (Fig. 7) shows that the solvent exposed surface area for hydrophobic residue types (C, V, I, M, L, $\mathrm{F}, \mathrm{W}$ and $\mathrm{Y}$ ) is increased by around $570 \AA^{2}$ compared to that of the crystal structure for the native peptide. ${ }^{13}$ The free energy cost of exposing such a hydrophobic surface area to solvent can be roughly estimated ${ }^{36}$ using a coefficient of $20 \mathrm{cal} \mathrm{mol}^{-1} \AA^{-2}$ ). This gives a free energy cost for exposure of $11.4 \mathrm{kcal} \mathrm{mol}^{-1}$. Given that the residues would not be completely shielded from 


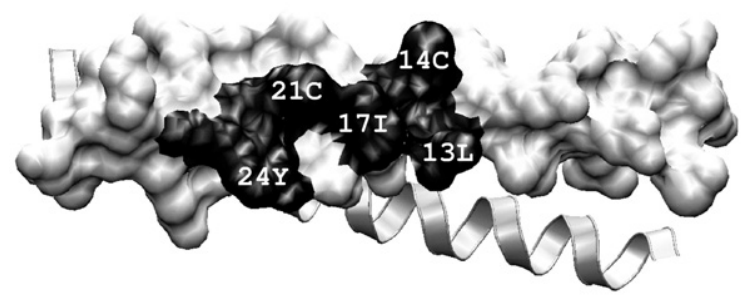

Fig. 7 The hydrophobic stripe created by the mutations introduced into $(i, i+7)$ Cys GCN4-p1. The model for the uncross-linked peptide incorporating the mutations was modelled with SYBYL from the crystal structure of GCN4-p1. ${ }^{13}$ For clarity the molecular surface of one of the monomers is shown in isolation with the other helix shown as a ribbon. The hydrophobic patch on the molecular surface created by the mutations is shown in black. Picture produced using VMD. ${ }^{21}$

solvent in an unfolded state, this provides an upper limit to the effect. This upper limit is comparable to the free energy of folding of GCN4-p1 $1^{31}$ of $-10.6 \mathrm{kcal} \mathrm{mol}^{-1}$. So if an unfolded state provides a degree of protection from solvent of the mutated residues a large effect on coiled coil formation could be expected.

An additional consideration is that the mutations have been introduced into the region of three helix-breaking residues (Asn16, Tyr17, and His18) that have been suggested to be important in the folding of the coiled coil. ${ }^{31}$ It is particularly unfortunate that two (Y17I and A24Y) of the four mutations were introduced as a consequence of the suggestion that the presence of a $\beta$-branched amino acid would enhance photo-control. ${ }^{8}$ This has now been shown not to be the case. ${ }^{9 a}$

The conjecture that the mutations created a stripe of hydrophobic amino acids could also account for the ability of the cross-linker to restore more native characteristics, as observed experimentally (Tables 1 and 3). The cross-linker modifies the two cysteine residues and is reasonably hydrophilic (containing two peptide moieties, Fig. 1). What is more, it can be expected to partly bury the Ile17 residue introduced below it (even in a partially folded peptide conformation). It can therefore be expected that cross-linking will have a large effect on any hydrophobic association event that involves the residues introduced by the mutations. We hypothesise that this effect may explain the promotion of helicity and coiled coil formation by the cross-linker (as seen by comparing values from Tables 1 and 3). In the case of the dark-adapted trans conformation, that was expected to be helix disrupting, it can be proposed that the prevention of unnatural hydrophobic interactions is the overriding factor.

In conclusion, we have produced a coiled coil peptide with a degree of photo-control of its helicity. However, by the injudicious choice of mutations to the cross-linker we have seriously altered the behaviour of the peptide. The current sequence is unsuitable for the ultimate aim of producing a photo-controlled GCN4-bZIP DNA binding protein. An advantage of the crosslinking method is that it can be applied in various ways. Future work aims at improvement, in particular by utilising the demonstrated ability of the cross-linker to control the helicity of peptides with an $i$ to $i+4$ inter-cysteine residue spacing. ${ }^{9}$ This offers a number of advantages, most notably that mutations can be made in a smaller area reducing the possibility of complicating effects. In addition the shorter spacing promises greater helix destabilisation in the dark-adapted trans crosslinker conformation as well as the potential to switch a larger proportion of the cross-linker into a cis conformation. ${ }^{9}$ We are confident that the development of photo-controlled variants of proteins will have many scientific applications.

\section{Acknowledgements}

We would like to acknowledge NSERC (Canada) (GAW), the Volkswagen Stiftung (Germany) (GAW) and the U.K. MRC and BBSRC Research Councils (grants G.4600017 and B/16000) (OSS). DGF was supported by a U.K. MRC Bioinformatics studentship. JRK was supported by a NSERC studentship. 


\section{References}

1 I. Willner and I. Rubin, Angew. Chem. Int. Ed. Engl., 1996, 35, 367.

2 L. Ulysse, J. Cubillos and J. Chmielewski, J. Am. Chem. Soc., 1995, 117, 8466.

3 R. Behrendt, C. Renner, M. Schenk, F. Wang, J. Wachtveitl, D. Oesterhelt and L. Moroder, Angew. Chem. Int. Ed. Engl., 1999, 38, 2771.

4 C. Renner, J. Cramer, R. Behrendt and L. Moroder, Biopolymers, 2000, 54, 501.

5 C. Renner, R. Behrendt, S. Sporlein, J. Wachtveitl and L. Moroder, Biopolymers, 2000, 54, 489.

6 R. Cerpa, F. E. Cohen and I. D. Kuntz, Fold. Des., 1996, 1, 91.

7 D. Liu, J. Karanicolas, C. Yu, Z. Zhang and G. A. Woolley, Bioorg. Med. Chem. Lett., 1997, 7, 2677.

8 J. R. Kumita, O. S. Smart and G. A. Woolley, Proc. Natl. Acad. Sci. U. S. A., 2000, 97, 3803.

9 (a) J. R. Kumita, D. G. Flint, O. S. Smart and G. A. Woolley, Protein Eng., 2002, in press; (b) D. G. Flint, J. R. Kumita, O. S. Smart and G. A. Woolley, Chem. Biol., 2002, 9, 391.

10 J. Wachtveitl, T. Nagele, B. Puell, W. Zinth, M. Kruger, S. Rudolph-Böhner, D. Oesterhelt and L. Moroder, J. Photochem. Photobiol. A, 1997, 105, 283.

11 M. Volk, Eur. J. Org. Chem., 2001, 2605.

12 W. D. Kohn, C. T. Mant and R. S. Hodges, J. Biol. Chem., 1997, 272, 2583.

13 E. O'Shea, J. D. Klemm, P. S. Kim and T. Alber, Science, 1991, 254, 539.

14 E. K. O’Shea, P. Rutkowski and P. S. Kim, Science, 1989, 243, 538.

15 R. S. Hodges, A. K. Saund, P. C. Chong, S. A. St-Pierre and R. E. Reid, J. Biol. Chem., 1981, $256,1214$.

16 S. Betz, R. Fairman, K. O' Neil, J. Lear and W. DeGrado, Philos. Trans. R. Soc. London, Ser. B, 1995, 348, 81.

17 T. E. Ellenberger, C. J. Brandl, K. Struhl and S. C. Harrison, Cell, 1992, 71, 1223.

18 L. Szilák, J. Moitra, D. Krylov and C. Vinson, Nature Struct. Biol., 1997, 4, 112.

19 A. M. Caamaño, M. E. Vázquez, J. Martínez-Costas, L. Castedo and J. L. Mascareñas, Angew. Chem. Int. Ed. Engl., 2000, 39, 3104.

20 S. J. Weiner, P. A. Kollman, D. A. Case, U. C. Singh, C. Ghio, G. Alagona, S. Profeta, Jr. and P. Weiner, J. Am. Chem. Soc., 1984, 106, 765.

21 W. F. Humphery, A. Dalke and K. Schulten, J. Mol. Graphics, 1996, 14, 33.

22 E. A. Merritt and D. J. Bacon, Methods Enzymol., 1997, 277, 505.

23 H. Edelhoch, Biochemistry, 1967, 6, 1948.

24 A. Bairoch and R. Apweiler, Nucleic Acids Res., 2000, 28, 45.

25 K. J. Lumb, C. M. Carr and P. S. Kim, Biochemistry, 1994, 33, 7361.

26 A. Kentsis and T. R. Sosnick, Biochemistry, 1998, 37, 14613.

27 T. R. Sosnick, S. Jackson, R. R. Wilk, S. W. Englander and W. F. DeGrado, Proteins: Struct., Funct., Genet., 1996, 24, 427.

28 A. Cammers Goodwin, T. J. Allen, S. L. Oslick, K. F. McClure, J. H. Lee and D. S. Kemp, J. Am. Chem. Soc., 1996, 118, 3082.

29 S. Y. M. Lau, A. K. Taneja and R. S. Hodges, J. Biol. Chem., 1984, 259, 3253.

30 S. Y. M. Lau, A. K. Taneja and R. S. Hodges, J. Chromatogr., 1984, 317, 129.

31 J. A. Zitzewitz, B. Ibarra-Molero, D. R. Fishel, K. L. Terry and C. R. Matthews, J. Mol. Biol., 2000, 296, 1105 .

32 K. T. O’Neil and W. F. DeGrado, Science, 1990, 250, 646.

33 N. E. Zhou, C. M. Kay and R. S. Hodges, Biochemistry, 1993, 32, 3178.

34 N. E. Zhou, C. M. Kay and R. S. Hodges, J. Mol. Biol., 1994, 237, 500.

35 S. D. Black and D. R. Mould, Anal. Biochem., 1991, 193, 72.

36 P. A. Karplus, Protein Sci., 1997, 6, 1302. 